\title{
THE GENERAL FORM OF THE ELASTIC STRESS AND DISPLACEMENT FIELDS OF THE FINITE CRACKED PLATE
}

\author{
Ali Ehsan Seif, Mohammad Zaman Kabir \\ Department of Civil Engineering, Amirkabir University of Technology (Tehran Polytechnic), Tehran, Iran \\ e-mail:seyf@aut.ac.ir; mzkabir@aut.ac.ir (corresponding author)
}

In this paper, the general forms of in-plane fields of a finite cracked plate have been achieved in the elastic range by expanding the potential functions of the infinite cracked plate about the crack tip. Subsequently, the stress intensity at the crack tip has been obtained. In addition, the numerical models have been provided by the finite element method.The effect of finite sizes on the stress and displacement fields has been detected and to validate the obtained analytical relations, the least-squares curve fitting has been used. Moreover, the calculated stress intensity correction factors have been compared with the existing results in the literature.

Keywords: finite cracked plate, least-squares curve fitting, general series form

\section{Introduction}

As Sadd (2005) explains, the stress singularity and displacement discontinuity are two characteristics of crack problems which lead to some complexity in solving these types of problems in the theory of elasticity. Solving many of crack problems will be done only by assuming the infinity of boundaries. Using this assumption, boundary conditions can be applied and the problem may be solvable. In the real problems with limited boundaries, usually, the results of infinite problems are used by applying a correction factor related to the finite size of the problem. The well known case is the use of geometry correction factor for the stress intensity at the crack tip in a finite cracked plate.

A wide range of studies using analytical and numerical methods are taken to determine the stress intensity correction factor or other stress and displacement fields at the crack tip. Isida (1971) estimated the value of the stress intensity at the crack tip by considering a special power series form for the stresses of finite cracked plates. Wu et al. used the weight function method to calculate the stress intensity under different loading conditions (Wu and Carlsson, 1983; Wu, 1984; Wu and Chen, 1989). Rice (1972), Ng and Lau (1999), Kiciak et al. (2003) and Jones et al. (2004) are further studies for the use of the weight function method in the crack problems. Many studies like Meng et al. (1998), Rangelova et al. (2003), Sahli et al. (2007) and Chen and Wang (2008) used numerical methods such as finite element and boundary element methods to observe the stress intensity or other fields at the crack-tip under different loading conditions.

Most of these studies examined only the near crack tip fields, especially the stress intensity factor and do not study the changes of stress and displacements at a distance far from the crack. Although there is no close form solution for the finite cracked plate, this study intends to extend the basic relations of infinite cracked plates and obtain the general power series form for the elastic stress and displacement fields of the finite crack plate so that it can be used for both near and far from the crack tip. To confirm the capability of the obtained analytical functions, some square plates with various crack lengths are modeled and analyzed with the finite element method. Then, the least-squares curve fitting is used to fit the analytical series functions to the numerically obtained stress and displacement profiles. 


\section{Analytical approach}

\subsection{Infinite cracked plate}

Figure 1 shows the central crack with length $2 a$ in an infinite plate which is under a uniform tension $\sigma_{o}$ perpendicular to the crack direction.

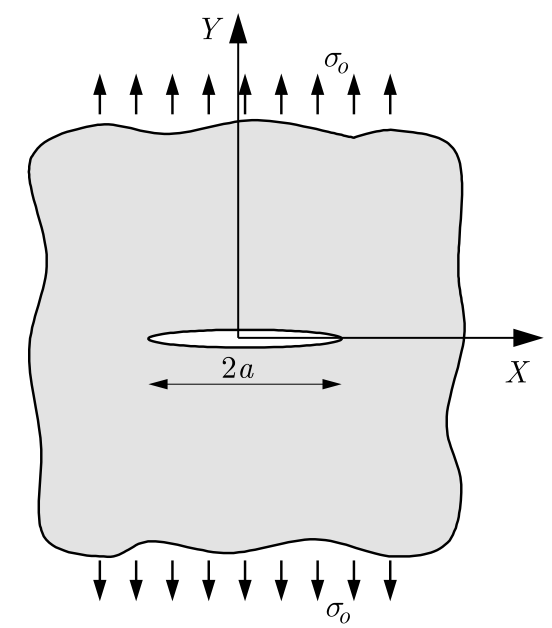

Fig. 1. Central cracked plate under tension

The classic solution of the infinite cracked plate using the complex variable method is available in many references such as Sadd (2005). Considering the complex coordinate $Z=X+\mathrm{i} Y$ and $\mathrm{i}=\sqrt{-1}$, two complex potential functions $\gamma$ and $\psi$ are used with the following relations for the elastic problem shown in Fig. 1

$$
\gamma(Z)=\frac{\sigma_{o}}{4}\left(2 \sqrt{Z^{2}-a^{2}}-Z\right) \quad \psi(Z)=\frac{\sigma_{o}}{4}\left(Z-\frac{a}{\sqrt{Z^{2}-a^{2}}}\right)
$$

Based on the potential functions, the stress combinations become

$$
\sigma_{y}+\sigma_{x}=2\left(\gamma^{\prime}+\bar{\gamma}^{\prime}\right) \quad \sigma_{y}-\sigma_{x}+2 \mathrm{i} \tau_{x y}=2\left(\bar{Z} \gamma^{\prime \prime}+\psi^{\prime}\right)
$$

where the prime and the over-bar signs denote derivative with respect to $Z$ and the complex conjugate, respectively, which results

$$
\sigma_{y}+\sigma_{x}=\sigma_{o} \operatorname{Re}\left(\frac{2 Z}{\sqrt{Z^{2}-a^{2}}}-1\right) \quad \sigma_{y}-\sigma_{x}+2 \mathrm{i} \tau_{x y}=\sigma_{o}\left[\frac{a^{2}(Z-\bar{Z})}{\sqrt{\left(Z^{2}-a^{2}\right)^{3}}}+1\right]
$$

in which Re is the real part. The corresponding displacements $U$ and $V$ respectively along $X$ and $Y$ can also be expressed by

$$
2 \mu(U+\mathrm{i} V)=k \gamma-Z \bar{\gamma}^{\prime}-\bar{\psi}
$$

or

$$
2 \mu(U+\mathrm{i} V)=\frac{\sigma_{o}}{4}\left[\frac{2\left(a^{2}-Z \bar{Z}\right)}{\sqrt{\left(\bar{Z}^{2}-a^{2}\right)^{3}}}+k\left(\sqrt{Z^{2}-a^{2}}-Z\right)+1-2 \bar{Z}\right]
$$

Since $E$ and $\nu$ are the modulus of elasticity and Poisson's ratio, $\mu=E / 2(1+\nu)$ and $k=(3-\nu) /(1+\nu)$ for plane stress problems. By setting $X$ and $Y$ in relations $(2.3)$ and (2.5), simpler forms of the stress and displacement can be obtained for specific cases. For example, for $Y=0$ and consequently $Z=\bar{Z}=X$, the value of the shear stress $\tau_{x y}$ is zero and the profiles of the normal stresses and displacements in the $X$ direction are expressed as follows: 
- for $X \leqslant a$

$$
\begin{aligned}
\sigma_{y} & =0 & \sigma_{x} & =-\sigma_{o} \\
V & =\frac{2 \sigma_{o}}{E} \sqrt{a^{2}-X^{2}} & U & =-\frac{\sigma_{o} X}{E}
\end{aligned}
$$

- for $X>a$

$$
\begin{array}{ll}
\sigma_{y}=\frac{\sigma_{o} X}{\sqrt{X^{2}-a^{2}}} & \sigma_{x}=\sigma_{y}-\sigma_{o} \\
V=0 & U=\frac{\sigma_{o}}{E}\left[(1-\nu) \sqrt{X^{2}-a^{2}}-X\right]
\end{array}
$$

Likewise, in the case $X=0$ we have $Z=-\bar{Z}=\mathrm{i} Y$, and hence

$$
\begin{aligned}
\sigma_{y} & =\frac{\sigma_{o} Y^{3}}{\sqrt{\left(Y^{2}+a^{2}\right)^{3}}} & \sigma_{x} & =\sigma_{o} \\
V & =\frac{\sigma_{o}}{E}\left[\frac{2 a^{2}+(1-\nu) Y^{2}}{\sqrt{a^{2}+Y^{2}}}+\nu Y\right] & U & =0
\end{aligned}
$$

Equations (2.6) to (2.8), which are obtained on the assumption of infinite dimensions, can be used for both near crack-tip and areas away from that. But in the real problems with finite sizes, the boundary conditions affect the stress and displacement fields. These effects become more, away from the crack tip and near the boundaries and they rule out the use of infinite plate relations for the finite one. In Fig. 2, the distribution of stresses $\sigma_{x x}$ and $\sigma_{y y}$ on the axes $X$ and $Y$ of a finite cracked plate are schematically compared with the infinite ones under the same tension stress $\sigma_{o}$.

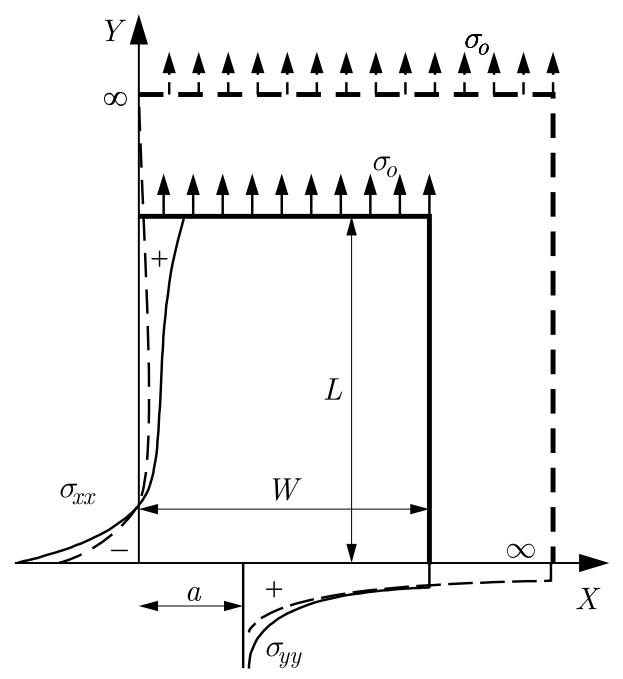

Fig. 2. Comparison of the stress distribution in one quarter of a finite cracked plate (solid line) and the infinite one (dash line) under the same tension; $(+)$ for tensile and $(-)$ for compressive stresses

\subsection{Finite cracked plate}

$\mathrm{Ng}$ and Lau (1999) used a method based on the expansion of field functions around the crack tip to get the general form of the stress intensity at the crack tip. In that method, using a variable transformation, the crack opening function $V$ from $(2.6)_{3}$ is expanded around the crack tip and consequently, the general form of the stress intensity is obtained as the infinite series. 
Present study uses the same method to obtain the general forms of all stresses and displacements around the crack tip and away from that. For this purpose, the variable transformation based on the parameters defined in Fig. 1 is

$$
t=Z^{2}-a^{2}
$$

so that at the crack tip we have $t=0$. Using (2.1) and the new variable $t$, let the general form of the potentials be taken as follows

$$
\gamma=\frac{\sigma_{o}}{4}\left[\omega(t)-B_{0} Z\right] \quad \psi=\frac{\sigma_{o}}{2}\left[B_{0} Z-\varphi(t)\right]
$$

where $\omega(t)$ and $\varphi(t)$ are two basic functions of $t$ and $B_{0}$ is an unknown coefficient. Comparing equations (2.1) with (2.10), it can be concluded that for the infinite cracked plate we have $B_{0}=1$, $\omega(t)=2 \sqrt{t}$ and $\varphi(t)=a^{2} / \sqrt{t}$. As Ng and Lau (1999) mentioned, according to the theory of linear elastic fracture mechanics, all the stresses and displacements in the vicinity of the crack tip are similar for any two cracks under arbitrary boundary conditions; thus the general form of the basic functions $\omega$ and $\varphi$ near the crack tip will be

$$
\omega_{N T}=2 A_{o} \sqrt{t} \quad \varphi_{N T}=\frac{A_{o} a^{2}}{\sqrt{t}}
$$

where subscript $N T$ denotes near tip. Subsequently, the normalized form of the basic functions with respect to the near tip can be defined as follows

$$
G(t)=\frac{\omega(t)}{\omega_{N T}} \quad F(t)=\frac{\varphi(t)}{\varphi_{N T}}
$$

Now, to reach the series form for basic functions, normalized forms (2.12) can be expanded in Taylor series about the crack tip $(t=0)$

$$
\begin{aligned}
& G(t)=G(0)+\frac{G^{(1)}(0)}{1 !} t+\frac{G^{(2)}(0)}{2 !} t^{2}+\cdots+\frac{G^{(n)}(0)}{n !} t^{n}+\cdots \\
& F(t)=F(0)+\frac{F^{(1)}(0)}{1 !} t+\frac{F^{(2)}(0)}{2 !} t^{2}+\cdots+\frac{F^{(n)}(0)}{n !} t^{n}+\cdots
\end{aligned}
$$

where $F(n)$ and $G(n)$ denote the $n$-th derivatives of $F$ and $G$ with respect to $t$ which are subject to the boundary conditions and the loading and, generally, can be used as the unknown coefficients in the series. It should be noted that approaching the crack tip, $G$ and $F$ are close to 1 so that at the crack tip $(t=0)$ we have $G(0)=F(0)=1$. Hence after simplifying $(2.13)$ and using (2.11) and (2.12), the general form of the basic functions would be

$$
\begin{aligned}
& \omega(t)=2 \sqrt{t}\left(A_{0}+A_{1} t+A_{2} t^{2}+\cdots+A_{n} t^{n}+\cdots\right)=\sum_{n=0}^{\infty} 2 A_{n} t^{n+1 / 2} \\
& \varphi(t)=\frac{a^{2}}{\sqrt{t}}\left(A_{0}+A_{1} t+A_{2} t^{2}+\cdots+A_{n} t^{n}+\cdots\right)=\sum_{n=0}^{\infty} a^{2} A_{n} t^{n-1 / 2}
\end{aligned}
$$

Finally, using (2.9) and substituting (2.14) respectively into (2.10), the general forms of the potentials would be expressed as follows

$$
\gamma=\frac{\sigma_{o}}{4}\left[\sum_{n=0}^{\infty} 2 A_{n}\left(Z^{2}-a^{2}\right)^{n+1 / 2}-B_{0} Z\right] \quad \psi=\frac{\sigma_{o}}{2}\left[B_{0} Z-\sum_{n=0}^{\infty} a^{2} A_{n}\left(Z^{2}-a^{2}\right)^{n-1 / 2}\right]
$$


Using above equations, the general forms of the stress and displacement fields can be achieved for the whole finite cracked plate with arbitrary boundary conditions. Substituting equations (2.15) into (2.2) and doing some mathematical works, each component of the stress can be expressed separately

$$
\begin{aligned}
& \sigma_{x}=\frac{\sigma_{o}}{2} \operatorname{Re}\left\{\sum_{n=0}^{\infty} A_{n} H_{n}\left[2 Z^{2}(Z-n \bar{Z})-a^{2}\left(\frac{2 n+3}{2 n+1} Z-\bar{Z}\right)\right]\right\}-B_{0} \sigma_{o} \\
& \sigma_{y}=\frac{\sigma_{o}}{2} \operatorname{Re}\left\{\sum_{n=0}^{\infty} A_{n} H_{n}\left[2 Z^{2}(Z+n \bar{Z})-a^{2}\left(\frac{6 n+1}{2 n+1} Z+\bar{Z}\right)\right]\right\} \\
& \tau_{x y}=\frac{\sigma_{o}}{2} \operatorname{Im}\left\{\sum_{n=0}^{\infty} A_{n} H_{n}\left[2 Z^{2}(n \bar{Z})-a^{2}\left(\frac{2 n-1}{2 n+1} Z+\bar{Z}\right)\right]\right\}
\end{aligned}
$$

where Re and Im are the real and imaginary parts, respectively, and

$$
H_{n}=(2 n+1)\left(Z^{2}-a^{2}\right)^{n-3 / 2}
$$

In the same way, the displacements are obtained in the series forms by using (2.15) and (2.4)

$$
\begin{aligned}
& U=\frac{\sigma_{o}}{4 \mu} \operatorname{Re}\left[\sum_{n=0}^{\infty} A_{n} J_{n}-B_{0}\left(\frac{k-1}{2} Z+\bar{Z}\right)\right] \\
& V=\frac{\sigma_{o}}{4 \mu} \operatorname{Im}\left[\sum_{n=0}^{\infty} A_{n} J_{n}-B_{0}\left(\frac{k-1}{2} Z+\bar{Z}\right)\right]
\end{aligned}
$$

in which

$$
J_{n}=k\left(Z^{2}-a^{2}\right)^{n+1 / 2}-\left[(2 n+1) Z \bar{Z}-a^{2}\right]\left(\bar{Z}^{2}-a^{2}\right)^{n-1 / 2}
$$

As it will be shown, equations (2.16) to (2.19) as general forms of the stresses and displacements can be used for finite cracked plates. It is necessary to note that the obtained equations are not limited to fields near the crack tip and can get stresses and displacements away from that and close to the boundaries. Although forms (2.16) to (2.19) are so complex, simplified equations can be obtained for special cases. For example, for $Y=0$, the profiles of non-zero stresses and displacements are expressed as follows:

- for $X \leqslant a$

$$
\begin{aligned}
& \sigma_{x}=-B_{0} \sigma_{o} \quad U=-\frac{\sigma_{o}}{E} B_{0} X \\
& V=\frac{2 \sigma_{o}}{E} \sum_{n=0}^{\infty} A_{n}(-1)^{n}\left(a^{2}-X^{2}\right)^{n-1 / 2}\left(a^{2}-X^{2}-\frac{1+\nu}{2} n X^{2}\right)
\end{aligned}
$$

- for $X>a$

$$
\begin{aligned}
& \sigma_{x}=\sigma_{o} \sum_{n=0}^{\infty} A_{n} X\left(X^{2}-a^{2}\right)^{n-3 / 2}\left[X^{2}-a^{2}-(2 n-1) n X^{2}\right]-B_{0} \sigma_{o} \\
& \sigma_{y}=\sigma_{o} \sum_{n=0}^{\infty} A_{n} X\left(X^{2}-a^{2}\right)^{n-3 / 2}\left[(4 n+1)\left(X^{2}-a^{2}\right)+(2 n-1) n X^{2}\right] \\
& U=\frac{\sigma_{o}}{E} \sum_{n=0}^{\infty} A_{n}\left(X^{2}-a^{2}\right)^{n-1 / 2}\left[(1-\nu)\left(X^{2}-a^{2}\right)-(1+\nu) n X^{2}\right]-\frac{\sigma_{o}}{E} B_{0} X
\end{aligned}
$$


Likewise, in the case $X=0$

$$
\begin{aligned}
& \sigma_{x}=\sigma_{o} \sum_{n=0}^{\infty} A_{n}(n+1)(-1)^{n} Y\left(Y^{2}+a^{2}\right)^{n-3 / 2}\left[2 a^{2}+(2 n+1) Y^{2}\right]-B_{0} \sigma_{o} \\
& \sigma_{y}=\sigma_{o} \sum_{n=0}^{\infty} A_{n}(-1)^{n} Y\left(Y^{2}+a^{2}\right)^{n-3 / 2}\left[2 n a^{2}-(2 n+1)(n-1) Y^{2}\right] \\
& V=\frac{\sigma_{o}}{E} \sum_{n=0}^{\infty} A_{n}(-1)^{n}\left(Y^{2}+a^{2}\right)^{n-1 / 2}\left[2 a^{2}+Y^{2}(1-\nu-n(1+\nu))\right]+\frac{\sigma_{o}}{E} B_{0} \nu Y
\end{aligned}
$$

\subsection{Calculation of the stress intensity}

As mentioned, based on the principles of linear elastic fracture mechanics theory, the stress and displacement fields near the crack tip have the same general forms for different boundary conditions; but their value is a function of the stress intensity at the crack tip. In other words, the stress intensity at the crack tip can be calculated from the fields of stress and displacements near the crack tip. With regard to the first mode of fracture, the stress intensity $K_{I}$ can be obtained from the stress field at $Y=0$ and $X \geqslant a$ as follows

$$
K_{I}=\lim _{r \rightarrow 0} \sigma_{y} \sqrt{2 \pi r} \quad r=X-a
$$

Now, using the dimensionless variable $x=X / a$, if $(2.21)_{2}$ is used with the above equation, it will be

$$
K_{I}=\lim _{x \rightarrow 1} \sigma_{o} \sqrt{\pi a} \sum_{n=0}^{\infty} \frac{A_{n} x}{\sqrt{x+1}}\left[(4 n+1)\left(x^{2}-1\right)^{n}+(2 n-1) n x^{2}\left(x^{2}-1\right)^{n-1}\right]
$$

or

$$
K_{I}=\sigma_{o} \sqrt{\pi a}\left[A_{0}(1)+A_{1}(3)+A_{2}(0)+\cdots+A_{n}(0)+\cdots\right]
$$

As can be seen, despite numerous terms in series $(2.21)_{2}$, the stress intensity at the crack tip is related to the first two terms of the series. In the field of linear fracture mechanics, for cracked problems with finite sizes, the so-called correction factor $\beta$ is defined as the dimensionless ratio of $K_{I} / \sigma_{o} \sqrt{\pi a}$ that includes the effect of boundary conditions and finite sizes. Hence, (2.25) can be rewritten as follows

$$
\beta=\frac{K_{I}}{\sigma_{o} \sqrt{\pi a}}=A_{0}+3 A_{1}
$$

Likewise, the stress intensity factor can be obtained based on the displacement field at $Y=0$ and $X \leqslant a$

$$
K_{I}=\lim _{r \rightarrow 0} \frac{E V}{4} \sqrt{\frac{2 \pi}{r}} \quad r=a-X
$$

in which $E$ is Young's modulus, and substituting $(2.20)_{3}$ into (2.27) we have

$$
\frac{K_{I}}{\sigma_{o} \sqrt{\pi a}}=\lim _{x \rightarrow 1} \sum_{n=0}^{\infty} \frac{A_{n} x}{\sqrt{x+1}}\left[(4 n+1)\left(x^{2}-1\right)^{n}+(2 n-1) n x^{2}\left(x^{2}-1\right)^{n-1}\right]
$$

and thus

$$
K_{I}=\sigma_{o} \sqrt{\pi a}\left[A_{0}(1)+A_{1}\left(\frac{1+\nu}{2}\right)+A_{2}(0)+\cdots+A_{n}(0)+\cdots\right]
$$


It should be noted that coefficients An in (2.25) and (2.29) are not necessarily identical, and each will be obtained independently by fitting the curves of stress and displacements. But the remarkable thing is that, as a result obtained by the stress field, this time also the stress intensity is related to the first two terms of the displacement filed series. In other words, the first two terms in series $(2.20)_{3}$ to $(2.22)_{3}$ or generally $(2.16)$ to $(2.18)$ are related to the fields near crack tip, and the other terms describe the effects of boundary conditions on the fields away from the crack tip. However, using (2.29), the correction factor will be obtained based on the displacement field as follows

$$
\beta=\frac{K_{I}}{\sigma_{o} \sqrt{\pi a}}=A_{0}+\frac{1+\nu}{2} A_{1}
$$

\section{Numerical modeling and verifications}

In order to assess the applicability of the analytical series for the finite cracked plate, the numerical models are provided in ABAQUS environment. In this context, the stress and displacement profiles have been extracted from the results of the prepared numerical models for specific cases discussed in Section 2 and using the technique of least-square curve fitting have been compared with the analytical results. Finally, the results of this comparison are presented in tables and charts. Figure 3 shows the geometric parameters of the finite cracked plate under uniaxial tensile load. Next, the description of the numerical models has been offered.

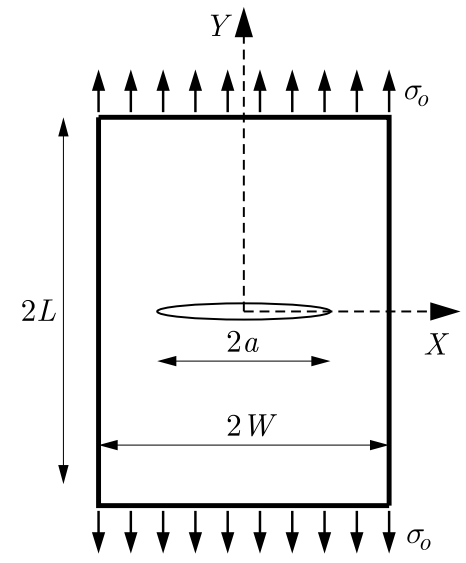

Fig. 3. The finite plate with a central cracked under tension

\subsection{Numerical modeling specifications}

The numerical models have been made in ABAQUS environment using 4-node doubly curved general-purpose shell elements S4R. After some mesh size sensitivity analysis, the proper finite element mesh with 3600 elements and an adequate refinement near the crack tips is illustrated in Fig. 4.

As can be seen from equations (2.23) to (2.30), the stress intensity is independent of the material properties $(E$ and $\nu)$ and the plate thickness $t$. The correction factor $\beta$ is also only related to the geometry of the plate. Therefore, several models of the square cracked plate $(L=W)$ have been developed with different aspect ratios $a / W=0.2 \sim 0.5$. It should be noted that in these models, the constant parameters are $E=200 \mathrm{GPa}, \nu=0.3, t=1 \mathrm{~mm}$, $W=200 \mathrm{~mm}$ and all exterior edges are free to move in the plane. 


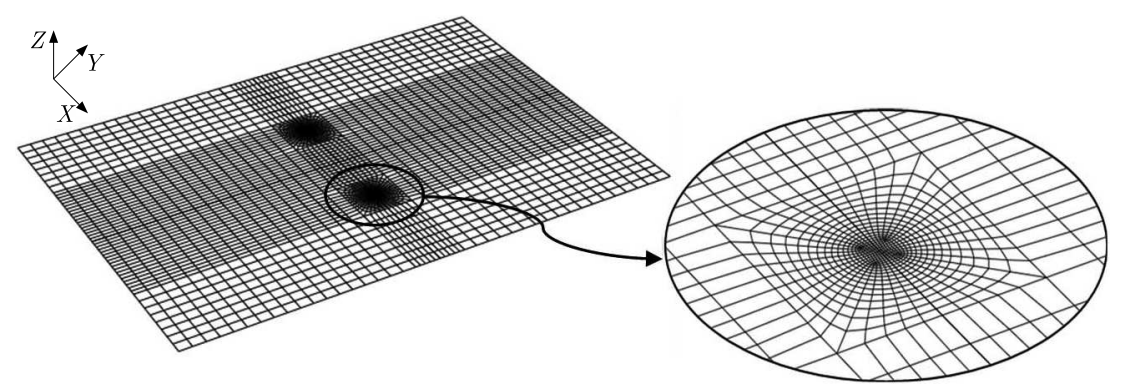

Fig. 4. Typical finite element mesh and crack tip detail

\subsection{Stress and displacement fields of the finite and infinite cracked plates}

Comparison of the normal stresses in the finite and infinite cracked plate is shown schematically in Fig. 2. In this Section, more complete and accurate comparison is made using the results of the finite element models. For this purpose, normalized curves of the stress and displacement profiles from the numerical models of the finite cracked plates are plotted against the existing theory relations of the infinite cracked plates in Figs. 5 to 8.

Figure 5 is related to the stresses $\sigma_{x}$ and $\sigma_{y}$ at $Y=0$ and $X \leqslant a$. As can be seen, the general form of the stress $\sigma_{x}$ for the finite cracked plates is similar to that of the infinite cracked plate that have been adapted to the conditions of zero stress at the free edges of the plate. But in the case of the stress $\sigma_{y}$, the limited size of the plate effectively changes the form of the curves. As expected, this effect is greater for plates with a larger relative crack length $a / W$. Moreover, with increasing crack length, the stress $\sigma_{y}$ is increased in areas near the crack tip which, based on the principles of fracture mechanics, it is affected by increasing the stress intensity. But away from the crack tip, that trend will be changed under the influence of boundary conditions so that the tensile stresses at the free edges of the plate are unexpectedly increased with the increasing relative crack length.
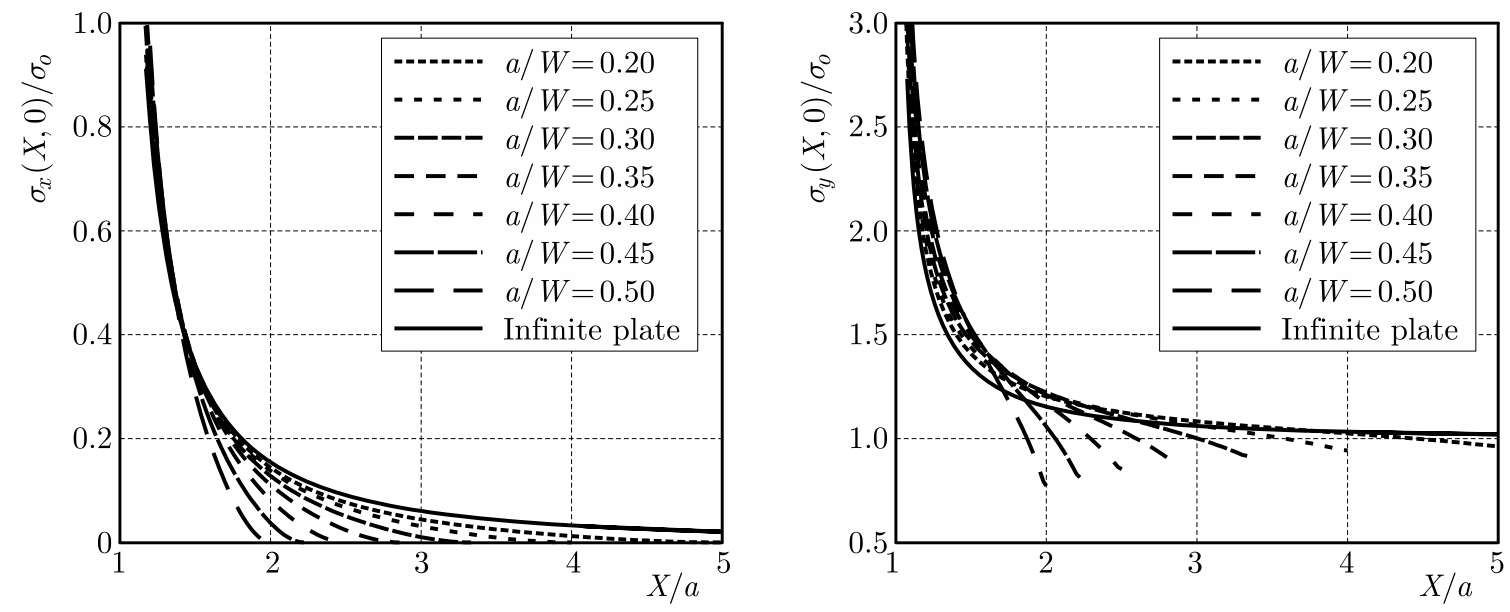

Fig. 5. Stress profiles at $Y=0$ and $X \geqslant a$ from numerical results of finite cracked plates (dash lines) in comparison with theoretical profiles of the infinite cracked plate (solid lines)

According to Fig. 6, the general form of the curves for the displacement $U$ in the finite cracked plates is similar to that of the infinite cracked plate in both linear and nonlinear parts. Likewise, in the case of crack opening displacement $V$, due to the remoteness of the free edges of the plates, the boundary conditions have the least impact on the general form of the curves. In terms of quantity, with the increasing relative crack length, the values of both displacements $U$ and $V$ are expectedly increased so that even in the normalized curves (with respect to the crack length), larger crack curves are above the smaller ones. 

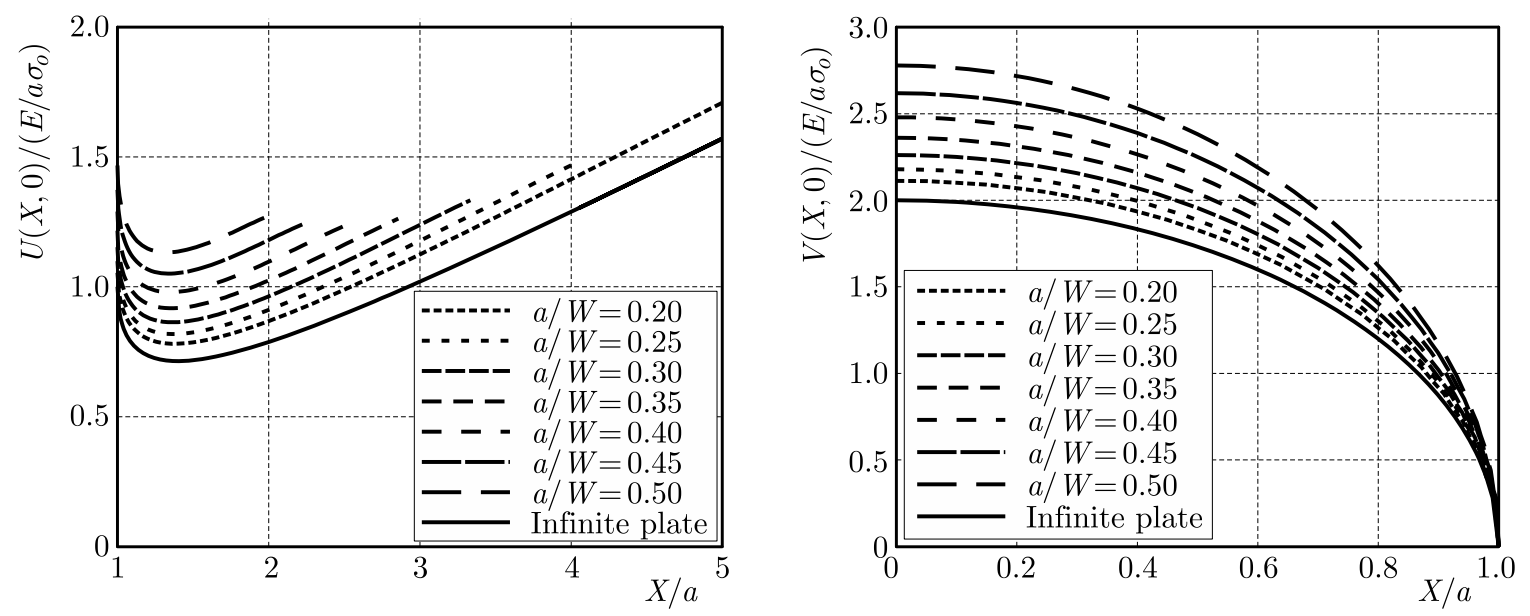

Fig. 6. Displacement profiles at $Y=0$ and $X \geqslant a$ from numerical results of finite cracked plates (dash lines) in comparison with theoretical profiles of the infinite cracked plate (solid lines)

Figure 7 shows the stresses $\sigma_{x}$ and $\sigma_{y}$ at $X=0$. In the case of stress $\sigma_{x}$, all curves show that the maximum compressive stress appears at the crack edge and decreases getting away from that. Subsequently, the compressive stress reaches zero at a distance of about $Y=0.8 a$ and then with a change of sign, it goes into the tensile phase. As can be seen, the maximum difference between the curves appears in the tensile phase so that for the infinite cracked plate, the curve is directed toward the edges and finally approaches zero; but the curves related to the finite cracked plates are upward near the edges and in most cases, the maximum tensile stress occurrs at the edge. In the case of stress $\sigma_{y}$, a significant difference between the overall trend of the curves related to the finite and infinite cracked plate cannot be viewed. The same result can be seen for the displacement $V$ at $X=0$ in Fig. 8. Comparison of all stress and displacement curves indicates that the finite sizes of the plate affect the general form of the stresses more than the displacements. This effect is more for the plates with a larger relative crack length and the maximum difference between the finite and infinite cracked plates occurs away from the crack tip and near the outer boundaries.
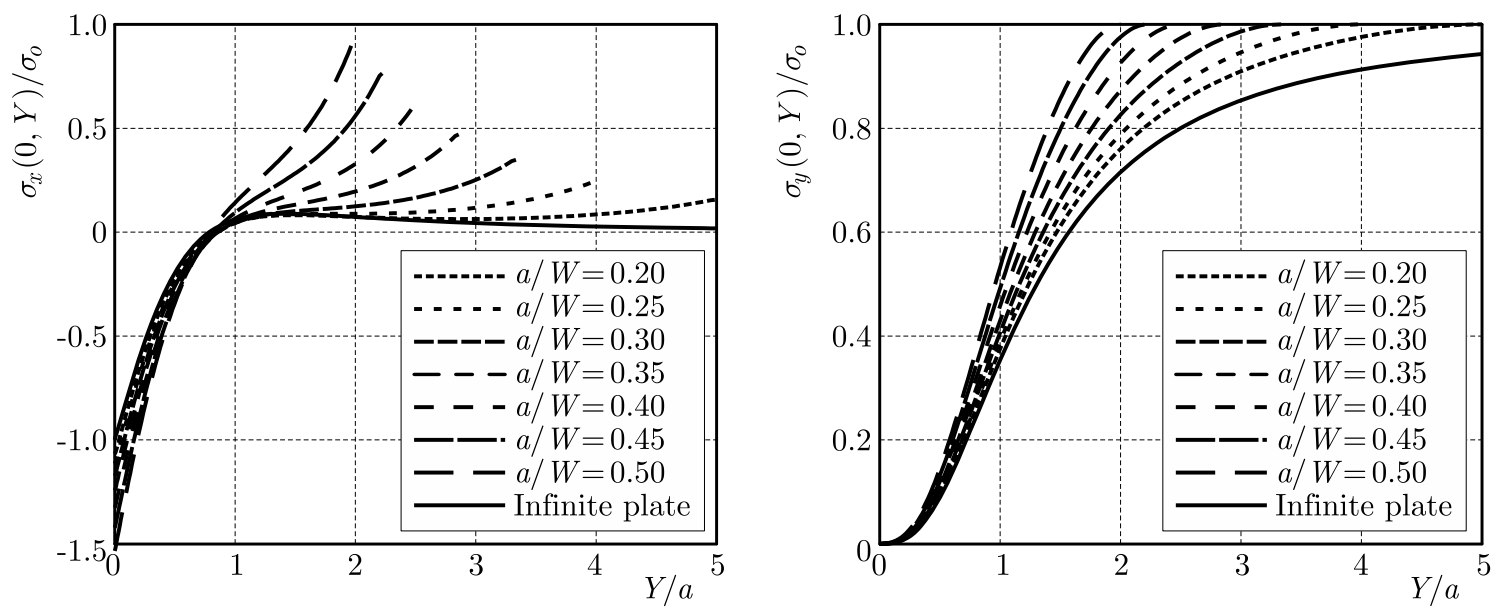

Fig. 7. Stress profiles at $X=0$ from numerical results of finite cracked plates (dash lines) in comparison with theoretical profiles of the infinite cracked plate (solid lines)

On the whole, it can be said that the theory relations of the infinite cracked plate cannot satisfy the boundary conditions of finite cracked plates. Also in terms of overall form of some in-plane fields, those relations have considerable differences with the results of the finite cracked 


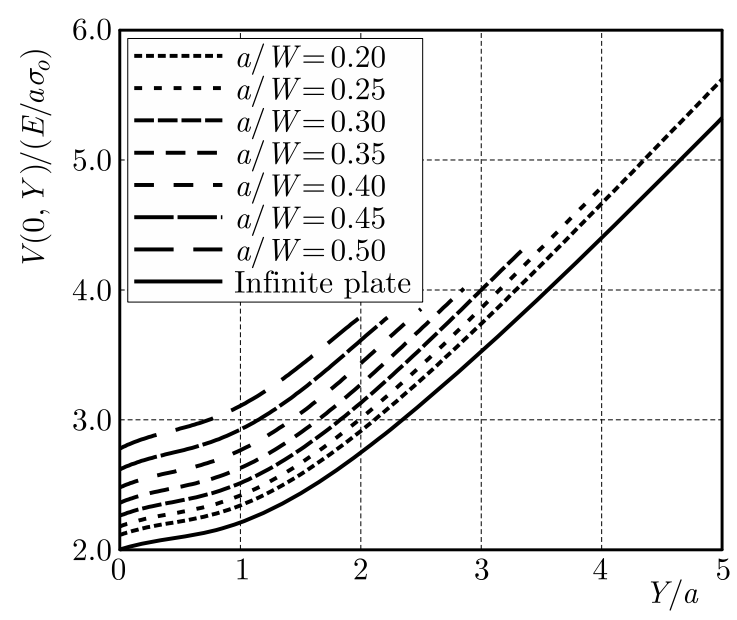

Fig. 8. Displacement profiles at $X=0$ from numerical results of finite cracked plates (dash lines) in comparison with theoretical profiles of the infinite cracked plate (solid lines)

plates. In this regard, the general forms of stresses and displacements fields are presented in Section 2.2 and their application for the finite cracked plates will be examined in the following Section.

\subsection{Validation of the analytical approach}

In order to validate the presented analytical approach, using the least-squares technique, the curves of the series obtained in Section 2.2 are fitted to the stress and displacement profiles derived from the numerical models for the finite plates. In the curve fitting, three relative crack lengths of $a / W=0.2,0.35,0.5$ are selected as small, medium and large cracks, and the minimum terms of the series are chosen so that so-called coefficient of determination $R^{2}$ with the precision of three decimal places is equal to 1. The results are presented in Figs. 9 to 12 and the curve fit parameters have been inserted into every curve, where $R^{2}$ is the coefficient of determination and $N$ is the number of series terms used in the curve fitting. It should be noted that during the curve fitting, the stress singularity at the tip of the crack can cause errors and reduce the accuracy of the results. Therefore, as shown in Fig. 9, the normalized stress fields $\sigma_{x}$ and $\sigma_{y}$ at $Y=0$ and $X \geqslant a$ are multiplied by the dimensionless term of $\sqrt{(X / a)^{2}-1}$ to avoid the errors and then the curve fitting has been done.

As can be seen, in all cases, the desired accuracy has been achieved only using a limited number of the series terms. The same results of displacement fields for this zone are shown in Fig. 10. By comparing Figs. 9 and 10, it can be seen that with the same accuracy in curve fitting, the number of required terms for displacement fields are always less than the number of terms needed for stress fields.

Figure 11 shows the curve fitting results of $\sigma_{x}$ and $\sigma_{y}$ at $X=0$. As noted in Section 3.2, in the tensile phase of stress $\sigma_{x}$, the overall trend of the curves related to the finite cracked plates are different from the theory relations of the infinite cracked plate; but as can be seen in Fig. 11, the obtained analytical series with only 3 terms are accountable for such a dissimilar trend and with a high accuracy fit on the numerical results achieved for the finite cracked plates. In total, for both stresses $\sigma_{x}$ and $\sigma_{y}$, using 3 terms of the series gives the desired accuracy. Figure 12 also represents the same results for the displacement fields at $X=0$.

\subsection{The stress intensity and correction factor}

In Section 2.3, the stress intensity and, consequently, correction factor are obtained based on the first two terms of the series related to the stress $\sigma_{y}$ and displacement $V$ at $Y=0$. In this Section, using the results of curve fitting, the correction factor has been attained from $(2.26)$ 

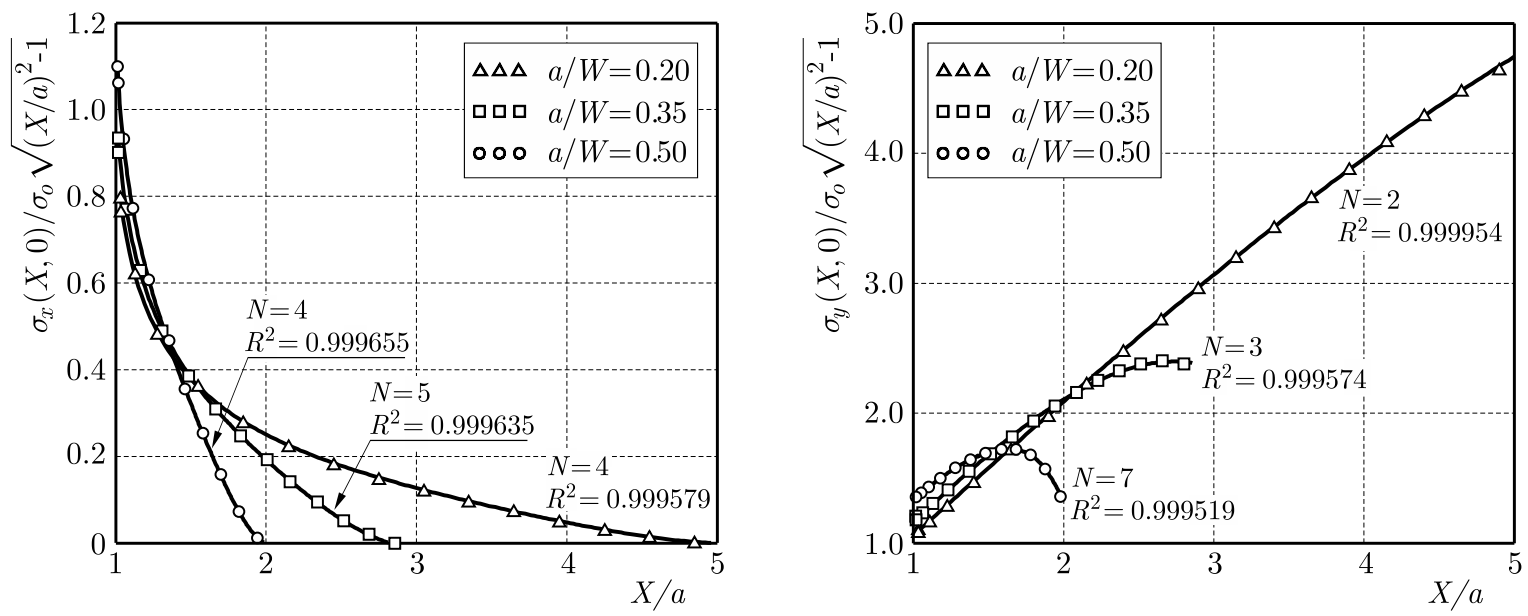

Fig. 9. Theoretical series curves (solid lines) related to stress fields at $Y=0$ and $X \geqslant a$ fitted to numerical results (symbols)
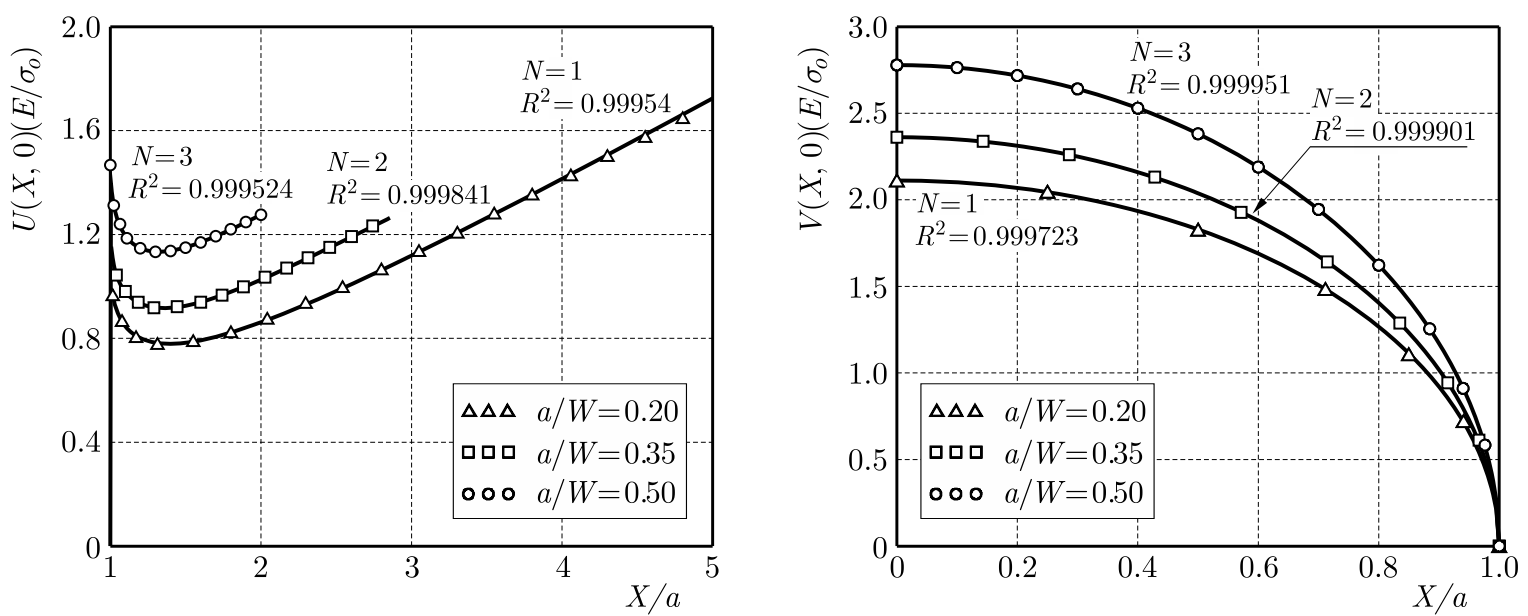

Fig. 10. Theoretical series curves (solid lines) related to displacement fields at $Y=0$ fitted to numerical results (symbols)
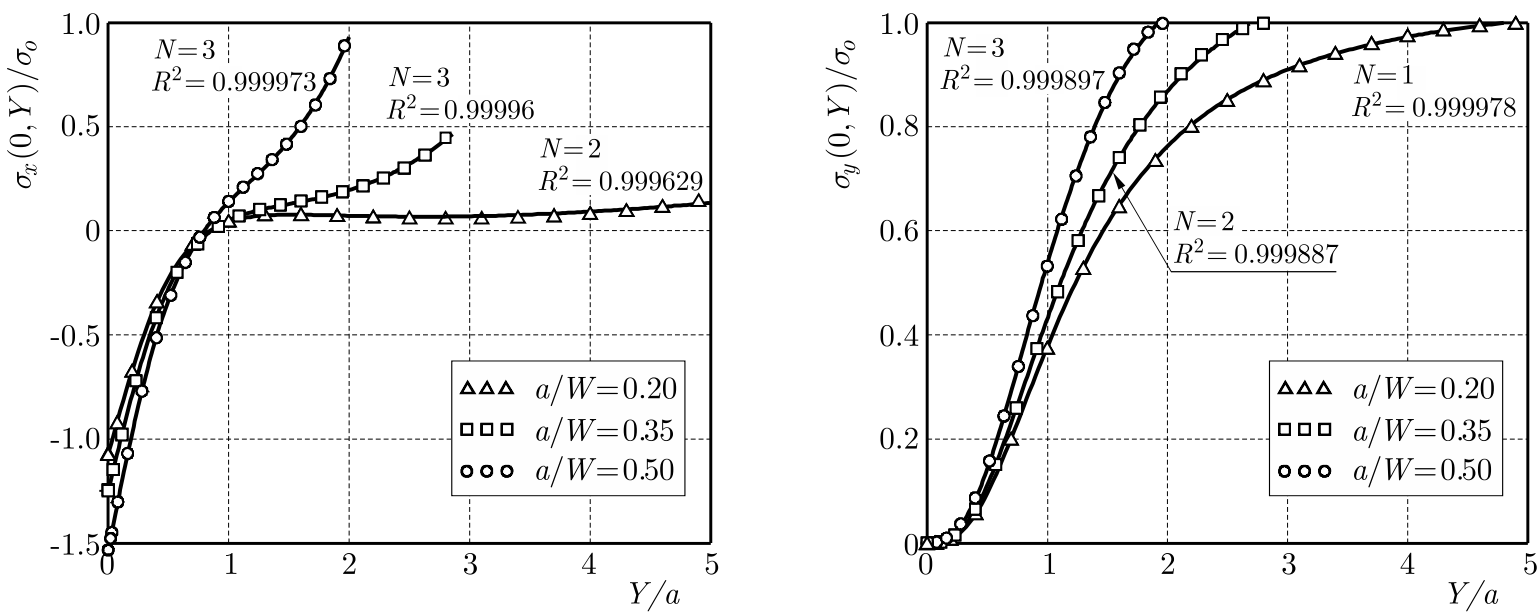

Fig. 11. Theoretical series curves (solid lines) related to stress fields at $X=0$ fitted to numerical results (symbols) 


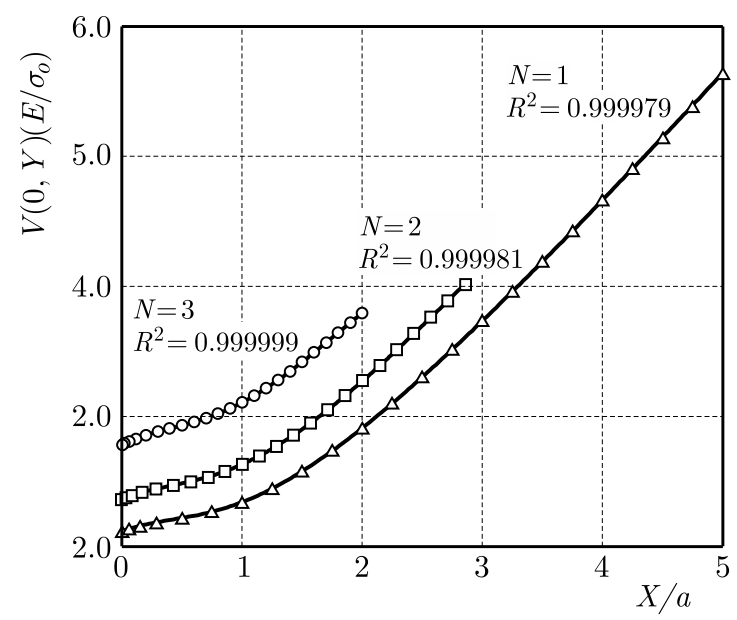

Fig. 12. Theoretical series curves (solid lines) related to displacement fields at $X=0$ fitted to numerical results (symbols)

and (2.30) and is presented in Table 1 for three relative crack lengths of $a / W=0.2,0.35,0.5$ in which the columns $A_{0}$ and $A_{1}$ contain the resulting coefficients of the curve fitting and the column $\beta$ represents the evaluated correction factors from (2.26) and (2.30). Moreover, in order to validate the results, the related correction factors from Isida (1971) are presented in the column $\beta_{R E F}$, and in the last column, the percentage difference between the results is provided.

Table 1. Correction factor from curve fitting process and the reference by Isida (1971)

\begin{tabular}{|c|c|c|c|c|c|c|}
\hline$a / W$ & Field & $A_{0}$ & $A_{1}$ & $\beta$ & $\beta_{R E F}$ & Error [\%] \\
\hline \hline 0.20 & $S_{y}(X, 0)$ & 1.05860 & -0.00076 & 1.05633 & 1.055 & 0.13 \\
\cline { 2 - 7 } & $V(X, 0)$ & 1.05496 & -0.00153 & 1.05397 & 1.055 & 0.10 \\
\hline \multirow{2}{*}{0.35} & $S_{y}(X, 0)$ & 1.18793 & -0.00762 & 1.16507 & 1.170 & 0.38 \\
\cline { 2 - 7 } & $V(X, 0)$ & 1.17125 & -0.00944 & 1.16511 & 1.170 & 0.38 \\
\hline \multirow{2}{*}{0.50} & $S_{y}(X, 0)$ & 1.41167 & -0.03556 & 1.30498 & 1.334 & 2.18 \\
\cline { 2 - 7 } & $V(X, 0)$ & 1.35431 & -0.03491 & 1.33162 & 1.334 & 0.18 \\
\hline
\end{tabular}

As can be seen, the analytical results of the present paper agree well with the results of Isida (1971). The maximum difference is $2.18 \%$. It is also observed that for different crack lengths, the calculated correction factors based on the displacement filed are always more accurate than the stress filed because firstly, the displacement at the crack tip is not singular as the stress field and secondly, in the finite element method, the obtained displacement fields are continuous at the boundaries of elements while there is no such continuity for the stress fields.

\section{Conclusion}

Since there is no closed form solution for finite cracked plates, in this paper, the general forms of stress and displacement fields of the finite cracked plate are achieved by expanding potential functions of an infinite cracked plate about the crack tip. In addition to analytical relations, numerical models of the finite cracked plate with different crack lengths have been provided in ABAQUS environment. Based on the numerical results, the stress and displacement profiles are plotted against the existing theory relations of infinite cracked plates. The plotted curves show that finite sizes of the plate affect the general form of stresses more than displacements, and the maximum difference between the finite and infinite cracked plates occurs away from the crack tip and near the outer boundaries. The observed difference indicates that the theory relations of 
the infinite cracked plate cannot satisfy the boundary conditions of the finite cracked plates and also in terms of overall form of some in-plane fields, those relations have considerable differences with the results of the finite cracked plates.

Accordingly, using the least-squares curve fitting, the obtained theoretical series for stresses and displacements of the finite cracked plates have been compared with the finite element results. The presented results indicate that the obtained analytical series with a limited number of terms are accountable for such a dissimilar trend and with a high accuracy fit to the numerical results achieved for the finite cracked plates. For example, the coefficient of determination $R^{2}$ with precision of three decimal places is equal to 1 by choosing only 7 terms of the series for singular stresses and 3 terms for other stress and displacement fields.

Moreover, by calculating the stress intensity on the basis of stress and displacement series, it has been shown that the stress intensity and the related correction factor $\beta$ are related to the first two terms of the series. Using the analytical relations with the results of curve fitting, the correction factors have been attained for some relative crack lengths and compared with the results of Isida (1971). The comparison shows that the analytical results of the present paper agree well with the results of Isida (1971), and the maximum difference is $2.18 \%$.

\section{References}

1. Chen C.H., Wang C.L., 2008, Stress intensity factors and T-stresses for offset double edge-cracked plates under mixed-mode loadings, International Journal of Fracture, 152, 149-162

2. IsIDA M., 1971, Effect of width and length on stress intensity factors of internally cracked plates under various boundary conditions, International Journal of Fracture Mechanics, 7, 301-316

3. Jones R., Peng D., Pitt S., Wallbrink C., 2004, Weight functions, CTOD, and related solutions for cracks at notches, Engineering Failure Analysis, 11, 79-114

4. Kiciak A., Glinka G., Burns D.J., 2003, Calculation of stress intensity factors and crack opening displacements for cracks subjected to complex stress fields, Journal of Pressure Vessel Technology, 125, 260-266

5. Meng G., Chen S., Liu H., Wang Z., 1998, The influence of plate size with double cracks on stress intensity factor, Communications in Numerical Methods In Engineering, 14, 429-436

6. NG S.W., LAU K.J., 1999, A new weight function expression for through cracks, Engineering Fracture Mechanics, 64, 515-537

7. Rangelova T., Dinevab P., Gross D., 2003, A hyper-singular traction boundary integral equation method for stress intensity factor computation in a finite cracked body, Engineering Analysis with Boundary Elements, 27, 9-21

8. RICE J.R., 1972, Some remarks on elastic crack-tip stress fields, International Journal of Solids and Structures, 8, 751-758

9. Sadd M.H., 2005, Elasticity: Theory, Applications and Numerics, Elsevier Inc., Oxford

10. Sahli A., Boutchicha D., Belarbi A., Rahmani O., 2007, Stress intensity solutions for cracked plates by the dual boundary method, Strength of Materials, 39, 5, 513-522

11. Wu X.R., Carlsson J., 1983, The generalised weight function method for crack problems with mixed boundary conditions, Journal of The Mechanics and Physics of Solids, 31, 6, 485-497

12. WU X.R., 1984, Approximate weight functions for center and edge cracks in finite bodies, Engineering Fracture Mechanics, 20, 1, 35-49

13. Wu X.R., Chen X.G., 1989, Wide-range weight function for center cracks, Engineering Fracture Mechanics, 33, 6, 877-886 